Doug Geisler, Eva K. Grebel, and Dante Minniti, eds.

\title{
Superbubble Metallicities and X-ray Luminosities
}

\author{
Sergiy Silich \\ Instituto Nacional de Astrofísica Optica y Electrónica, AP 51 y 216, \\ 72000 Puebla, México \\ Sally Oey \\ Lowell Observatory, 1400 West Mars Hill Rd., Flagstaff, AZ 86001, \\ $U S A$
}

\begin{abstract}
We show that X-ray emission and dynamical properties of superbubbles around OB associations are affected by metal ejection from the enclosed Type II supernovae (SNe). The $\mathrm{SN}$ and massive star yields may significantly change the superbubble interior metallicity and enhance its X-ray luminosity.
\end{abstract}

\section{Introduction}

Energy deposition by stellar winds and SN explosions within OB associations generates strong shocks which collect interstellar medium (ISM) into thin shells having gaseous cavities with characteristic sizes of several dozen to a few hundred pc. This mechanical feedback determines the large-scale structure and thermal state of interstellar gas, and therefore is fundamental to understanding the origin and time evolution of both cold and hot components of the ISM.

The LMC harbors a wealth of superbubbles, many of which have been detected in soft X-rays (Chu \& Mac Low 1990; hereafter CM90). Their dynamical properties have been studied by Oey (1996), who revealed two subsets of objects: high and low velocity superbubbles. The comparison of the observed Xray luminosities with the model predictions revealed that some X-ray bright superbubbles have $\mathrm{X}$-ray luminosities up to an order of magnitude higher than the expected values (CM90; Wang \& Helfand 1991).

Two models have been proposed to resolve this discrepancy: off-center SN explosions (CM90) and mass-loading by dense cloud destruction (Arthur \& Henney 1996). However, to date no firm evidence has been found to confirm directly either of these hypotheses. Here we examine another possibility, namely time variation in the metallicity of the $\mathrm{X}$-ray emitting gas, that results from metal ejection by $\mathrm{SN}$ explosions of the parent OB-association.

\section{Hot gas metallicity and bubble $\mathrm{X}$-ray emission}

We model the metallicity of the hot superbubble interior following Silich et al. (2001). This employs the Maeder (1992) stellar evolution models and a numerical 

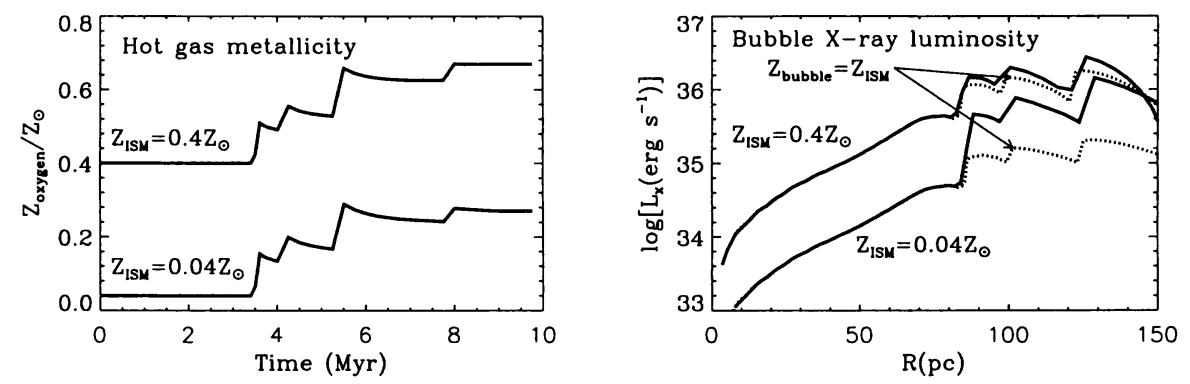

Figure 1. The evolution of bubble metallicity and X-ray luminosity. Dotted lines show the standard model's predictions.

scheme based on the thin layer approximation by Bisnovatyi-Kogan \& Silich (1995). As an example, we use the stellar contents for the LMC superbubble DEM L50 found by Oey (1996). We assume that massive star and SN products are well mixed with the gas evaporated from the cold radiative shell. This effectively changes the inner hot gas metallicity and thus modifies the modelpredicted X-ray emission.

We use the energy input rate derived for DEM L50, which includes contributions from two unobserved SN progenitors whose existence is inferred from a Salpeter IMF (Oey 1996), and assume a homogeneous ambient ISM with $\mathrm{n}_{\text {ism }}=1.4 \mathrm{~cm}^{-3}$. Figure 1 shows results for two models, which represent shell evolution into ambient ISM metallicities of $Z_{i s m}=0.4 Z_{\odot}$ and $0.04 Z_{\odot}$.

\section{Conclusions}

Our results imply that, if the host galaxy metal abundance is well below so$\operatorname{lar}\left(Z_{i s m}<0.1 Z_{\odot}\right)$, then enrichment by massive star products may completely dominate the interior chemical composition soon after the onset of SN explosions. This enhances superbubble X-ray luminosities and predicts only weak dependence on the parent galaxy metallicity.

\section{References}

Arthur, S. J. \& Henney, W. 1996, ApJ, 457, 752

Bisnovatyi-Kogan G.S. \& Silich S.A. 1995, RevModPhys, 67, 661

Chu Y.-H. \& Mac Low M.-M. 1990, ApJ, 365, 510

Maeder, A. 1992, A\&A,264, 105

Oey, M. S. 1996, ApJ, 467, 666

Silich, S.A., Tenorio-Tagle, G., Terlevich R., Terlevich E., \& Netzer H. 2001, MNRAS, 324, 191

Wang, Q. D. \& Helfand, D. J. 1991, ApJ, 373, 497 